\title{
Comparative transcriptome analysis of roots, stems, and leaves of Pueraria lobata (Willd.) Ohwi: identification of genes involved in isoflavonoid biosynthesis
}

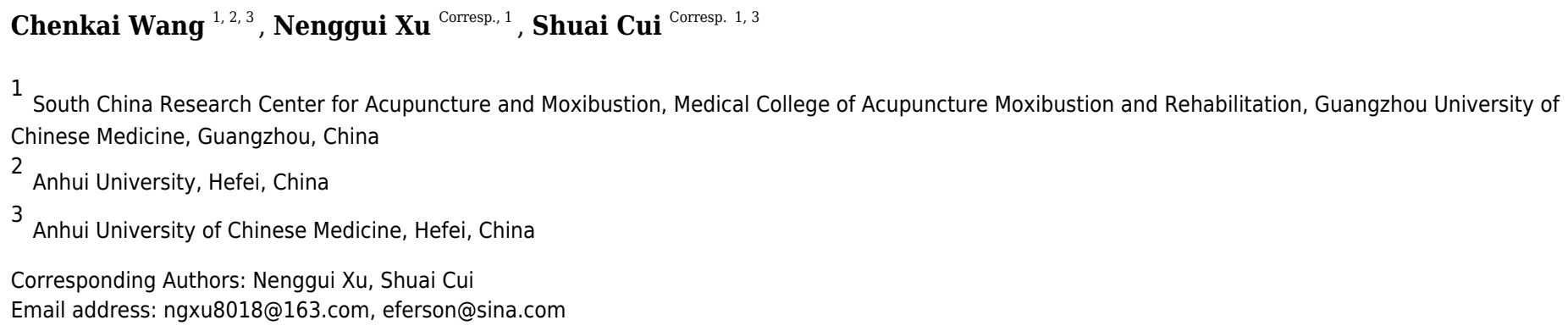

Background. Pueraria lobata (Willd.) Ohwi is a valuable herb used in traditional Chinese medicine. Isoflavonoids are the major bioactive compounds in $P$. lobata, namely puerarin, daidzin, glycitin, genistin, daidzein, and glycitein, which have pharmacological properties of anti-cardiovascular, anti-hypertension, anti-inflammatory, and anti-arrhythmic.

Methods. To characterize the corresponding genes of the compounds in the isoflavonoid pathway, RNA sequencing (RNA-Seq) analyses of roots, stems, and leaves of $P$. lobata were carried out on the BGISEQ-500 sequencing platform. Results. We identified 140,905 unigenes in total, of which 109,687 were annotated in public databases, after assembling the transcripts from all three tissues. Multiple genes encoding key enzymes, such as IF7GT and transcription factors, associated with isoflavonoid biosynthesis were identified and then further analyzed. Quantitative real-time PCR (qRT-PCR) results of some genes encoding key enzymes were consistent with our RNA-Seq analysis. Differentially expressed genes (DEGs) were determined by analyzing the expression profiles of roots compared with other tissues (leaves and stems). This analysis revealed numerous DEGs that were either uniquely expressed or up-regulated in the roots. Finally, quantitative analyses of isoflavonoid metabolites occurring in the three $P$. lobata tissue types were done via highperformance liquid-chromatography and tandem mass spectrometry methodology (HPLCMS/MS). Our comprehensive transcriptome investigation substantially expands the genomic resources of $P$. lobata and provides valuable knowledge on both gene expression regulation and promising candidate genes that are involved in plant isoflavonoid pathways. 


\section{Comparative transcriptome analysis of roots, stems, and}

\section{2 leaves of Pueraria lobata (Willd.) Ohwi: identification of 3 genes involved in isoflavonoid biosynthesis}

4 Chenkai Wang ${ }^{1,2,3}$, Nenggui $\mathrm{Xu}^{1^{*}}$, Shuai $\mathrm{Cui}^{1^{*}}$

$5 \quad{ }^{1}$ South China Research Center for Acupuncture and Moxibustion, Medical College of Acupuncture

6 Moxibustion and Rehabilitation, Guangzhou University of Chinese Medicine, 510006 Guangzhou, China

$7 \quad{ }^{2}$ Anhui University, Hefei, China

$8 \quad{ }^{3}$ Anhui University of Chinese Medicine, Hefei 230038, China

9 * Correspondence: ngxu8018@163.com; eferson@sina.com

10 ABSTRACT

Background. Pueraria lobata (Willd.) Ohwi is a valuable herb used in traditional Chinese medicine. Isoflavonoids are the major bioactive compounds in P. lobata, namely puerarin, daidzin, glycitin, genistin, daidzein, and glycitein, which have pharmacological properties of anti-cardiovascular , anti-hypertension, antiinflammatory, and anti-arrhythmic.

Methods. To characterize the corresponding genes of the compounds in the isoflavonoid pathway, RNA sequencing (RNA-Seq) analyses of roots, stems, and leaves of $P$. lobata were carried out on the BGISEQ-500 sequencing platform.

Results. We identified 140,905 unigenes in total, of which 109,687 were annotated in public databases, after assembling the transcripts from all three tissues. Multiple genes encoding key enzymes, such as IF7GT and transcription factors, associated with isoflavonoid biosynthesis were identified and then further analyzed. Quantitative real-time PCR (qRT-PCR) results of some genes encoding key enzymes were consistent with our RNA-Seq analysis. Differentially expressed genes (DEGs) were determined by analyzing the expression profiles of roots compared with other tissues (leaves and stems). This analysis revealed numerous DEGs that were either uniquely expressed or up-regulated in the roots. Finally, quantitative analyses of isoflavonoid metabolites occurring in the three $P$. lobata tissue types were done via high-performance liquidchromatography and tandem mass spectrometry methodology (HPLC-MS/MS). Our comprehensive transcriptome investigation substantially expands the genomic resources of $P$. lobata and provides valuable knowledge on both gene expression regulation and promising candidate genes that are involved in plant 
isoflavonoid pathways.

Keywords Pueraria lobata (Willd.) Ohwi, Transcriptome, RNA sequencing, Isoflavonoid biosynthesis, Differentially expressed genes

\section{INTRODUCTION}

The species Pueraria lobata (Willd.) Ohwi (P. lobata), commonly known as 'Kudzu', is a medical and edible plant in China that is widely distributed in temperate regions of China, Korea, Japan, and India(Chen et al. 2018; Jin et al. 2012). The dried root of $P$. lobata accumulates abundant isoflavonoids including puerarin, daidzein, daidzin, and genistein(He et al. 2011; Wong et al. 2011), and it has pharmacological activity for treating alcoholism, antioxidant, and other illnesses(Carai et al. 2000; Miyazawa et al. 2001). Puerarin is considered to be the main active isoflavonoid of P. Radix and P. lobata(Du et al. 2010; Ohshima et al. 1988; Wang et al. 2017), which reportedly has bioactivity against cardiovascular disease(Pan et al. 2011), vascular hypertension(Han et al. 2015) and it can improve insulin sensitivity(Meezan et al. 2005). Isoflavonoid synthesis is a branch of the flavonoid pathway. In the upstream pathway, the conversion of phenylalanine to liquiritigenin and naringenin happens through successive actions of phenylalanine ammonia-lyase (PAL), trans-cinnamate 4-monooxygenase $(\mathrm{C} 4 \mathrm{H})$, 4-coumarate-CoA ligase $(4 \mathrm{CL})$, chalcone synthase $(\mathrm{CHS})$, and chalcone isomerase (CHI)(Deavours \& Dixon 2005; Vogt 2010). In the downstream pathway, isoflavonoids are synthesized from liquiritigenin or naringenin under the hydroxylation, methylation, and glycosylation by 2hydroxyisoflavanone synthase (IFS), 2-hydroxyisoflavanone dehydratase (HIDH), methyltransferases and glycosyltransferases, respectively(Li et al. 2016; Wang et al. 2017).

However, the study of molecular biology of $P$. lobata is relatively limited and only 6,365 ESTs sequences are available from the NCBI database(He et al. 2011). The lack of reference genome information of P. lobata plant hinders the further studies on the underlying genes involved in essential biological processes related to isoflavonoid biosynthesis. Additionally, TFs potentially involved in isoflavonoid biosynthesis pathway in $P$. lobata have not been reported except the research on isoflavonoid-related TFs in P. thomsonii (He et al. 2019).

RNA sequencing (RNA-Seq) analysis has been shown to be an efficient methodology for functional gene discovery and the identification of secondary metabolite pathways in those non-model plant species lacking known genomic sequences(Garg \& Jain 2013). Therefore, RNA-Seq is a powerful tool for the capturing of both coding and non-coding sequences, the mining functional genes and the quantification of gene expression levels(Ozsolak \& Milos 2011; Wang et al. 2009; Zhang et al. 2018). At present, this approach was successfully used for transcriptome sequencing of some other medicinal plants, such as Zanthoxylum planispinum(Kim et al. 2019), Trachyspermum ammi(Soltani Howyzeh et al. 2018), Picrorhiza kurroa(Vashisht et al. 2016), and Salvia miltiorrhiza(Chang et al. 2019). 
In this study, we performed a deep transcriptome analysis of three different tissues (roots, stems, and leaves) of $P$. lobata plants and identified the structural genes and transcription factors (TFs) potentially involved in isoflavonoid biosynthesis. This study forms the basis for further exploration of molecular mechanisms of isoflavonoid biosynthesis in P. lobata.

\section{MATERIALS \& METHODS}

\section{Plant material and RNA extraction}

The materials (roots, leaves, and stems) of P. lobata were collected, in August 2018, from the town of Huaiyuan, Tongbai County, and Nanyang City, all located in Henan Province, China, with verbal permission of the Manager Hongchao Shi (Guangzhou Baiyunshan Chen Liji Pharmaceutical Factory Co., Ltd.), and authenticated by Professor Dequn Wang (Anhui University of Chinese Medicine). Separated tissues of three individual plants were immediately frozen in liquid nitrogen and stored at $-80^{\circ} \mathrm{C}$ until their respective extraction of RNA. Total RNA was extracted from each of the three tissues (roots, leaves, stems) of P. lobata plants in three replicates, by using the Spectrum Plant RNA Kit (Aidlab Biotech, Beijing, China), and following the manufacturer's instructions. The quality and quantity of total RNA were respectively analyzed using the NanoDrop spectrophotometer and Agilent 2100 Bioanalyzer (Thermo Fisher Scientific,Waltham, MA, USA) (Supplementary Table S1).

\section{Isoflavonoid extraction and HPLC-MS/MS determination}

For the isoflavonoid quantifications, $0.5 \mathrm{~g}$ of dried $P$. lobata tissues (roots, leaves, or stems) were directly extracted with $40 \mathrm{ml}$ of methanol by ultrasonication for $20 \mathrm{~min}$ and then centrifuged at 10,000 rpm for $10 \mathrm{~min}$. The supernatant was obtained for the following HPLC-MS/ MS analysis. Puerarin, daidzin, glycitin, genistin, genistein, daidzein, glycitein, and formononetin standards were obtained from Shanghai Yuanye Biological Technology Co., Ltd (Shanghai, China); for each, a standard calibration curve was established at concentrations of $0.1 \mathrm{mg} / \mathrm{L}, 0.2 \mathrm{mg} / \mathrm{L}, 0.5 \mathrm{mg} / \mathrm{L}, 1 \mathrm{mg} / \mathrm{L}$, and 2 mg/L, respectively (Supplementary Fig.1). The HPLC-MS/MS system (Acquity, Waters, Milford, MA, USA) was used to analyze isoflavonoid metabolomics. Chromatographic separations of all samples were performed on Agilent ZORBAX Eclipse Plus C18 (dimensions: $2.1 \mathrm{~mm} \times 100 \mathrm{~mm}, 1.8 \mu \mathrm{m}$ particle size), using a gradient mixture of water (solvent A) and acetonitrile (solvent B) as the mobile phase. The column's temperature was controlled at $35^{\circ} \mathrm{C}$ and the flow rate set to $0.3 \mathrm{~mL} / \mathrm{min}$. The gradient conditions were optimized as follows: 0 30 $\mathrm{min}, 5 \% \sim 25 \% \mathrm{~B} ; 30 \sim 40 \mathrm{~min}, 25 \% \sim 45 \% \mathrm{~B} ; 40.01 \sim 45 \mathrm{~min}, 5 \% \mathrm{~B}$. The sample injection volume was 10 $\mu \mathrm{L}$. Mass spectrometry was carried out with an Agilent LC/MS/MS 6460 mass spectrometer (Agilent Technologies Inc., Santa Clara, CA, USA) in negative ion mode with an ESI source. The optimized conditions 
93

94

for the mass spectrometer were as follows: ESI source voltage of $3.5 \mathrm{kV}$; nebulization with nitrogen, at 40 psi; nebulizer flow at $8.0 \mathrm{~L} / \mathrm{min}$, and a temperature of $350{ }^{\circ} \mathrm{C}$. The scan range of mass spectra was set to $50-2000$ $\mathrm{m} / \mathrm{z}$, and ultra-high pure helium (He) served as the collision gas. The optimized MS parameters of isoflavonoid compounds can be found in Supplementary Table S2.

\section{Library construction and RNA sequencing}

The total RNA isolated from each plant tissue sample was applied to RNA-Seq library preparation, by following the protocol described in(Zhu et al. 2018). Briefly, total RNA was first treated with DNase I (TaKaRa, China) to remove all traces of genomic DNA; next, the mRNA molecules were enriched using oligo(dT)-attached magnetic beads and fragmented into smaller pieces by applying divalent cations, followed by their first-strand and second-strand cDNA synthesis. Then, these cDNA fragments were subjected to endrepair and 3'adenylation and ligated to adapters, after which they were PCR-amplified to generate the final cDNA library. The single-stranded circular DNA was formatted as the final cDNA library for evaluation in the Agilent 2100 Bioanalyzer (ABI, New York, NY, USA), and later sequencing on the BGISEQ-500 platform (Beijing Genomics Institute, Wuhan, China).

\section{Transcriptome assembly}

High-quality reads were obtained by discarding any reads with adaptors, unknown nucleotides ( $>5 \%)$ and those of low quality using the software tool SOAPnuke (Chen et al. 2017) (v1.4.0; parameters: -1 5 -q 0.5 -n 0.1 ) and Trimmomatic (v0.36; parameters: ILLUMINACLIP:2:30:10 LEADING:3 TRAILING: 3 SLIDINGWINDOW: 4:15 MINLEN:50). Since a reference genome for $P$. lobata was lacking, the high-quality reads obtained were assembled de novo by using Trinity(Grabherr et al. 2013) (v2.8.4). The assembled transcripts were then grouped using the TGI clustering tool (TGICL)(Pertea et al. 2003), discarding redundant sequences and retaining the non-redundant sequences, the latter termed unigenes.

\section{Functional annotation and differential expression analysis}

Functional annotation was done by comparing homologous sequences against the UniProt databases using BLASTX(Yang et al. 2014) with an E-value threshold of 1.0e-5. Also searched in this way were the databases of NR (non-redundant protein sequence), NT (nucleotide), KOG (clusters of euKaryotic Orthologous Groups), KEGG (Kyoto Encyclopedia of Genes and Genome), and SwissProt (a manually annotated and reviewed protein sequence database). The GO (Gene Ontology) annotation was carried out using Blast2GO (v2.5.0)(Conesa \& Götz 2008), and the sequences searched for in the Pfam (protein families) with the 
124

125

126

127

128

129

130

131

132

133

134

135

136

137

138

139

140

141

142

143

144

145

146

147

148

149

150

151

\section{RESULTS}

Hmmscan tool (v3.0) set to its default parameters.

To identify differentially expressed mRNAs in roots compared to leaves or stems, clean transcriptome reads of each sample were mapped onto the genome sequence of P. lobata using Bowtie2 (v2.2.5)(Langmead \& Salzberg 2012). The expression levels of unigenes were calculated using the RSEM (v1.2.8)(Li \& Dewey 2011) software program; those unigenes with a fold-change (FC) $\geq 2.00$ and FDR (false discovery rate) $\leq 0.001$ were designated as DEGs (differentially expressed genes) by the PoissonDis method(Dembélé \& Kastner 2014; Kim \& van de Wiel 2008).

Multiple sequence alignment was conducted with Clustalx v2.0 software. Conserved domains in the amino acid sequence of $P$. lobata IF7GT were identified via the Conserved Domains Database (http://www.ncbi.nlm.nih.gov/Structure/cdd/wrpsb.cgi/). The secondary structure of P. lobata IF7GT was predicted using the SWISS-MODEL(Bertoni et al. 2017) and ESPript v3.0 software(Robert \& Gouet 2014).

\section{Analysis of transcription factor (TF)}

The respective ORF of unigenes in the transcriptome of $P$. lobata root, leaf, and stem tissues was detected by Getorf software(Rice et al. 2000). Each was then mapped to the plant TF database (PlantTFDB) by Hmmsearch(Mistry et al. 2013) using BLASTX (E-value $\leq 1 \mathrm{e}-5$ ).

\section{Quantitative real-time PCR (qRT-PCR) analysis}

To validate the transcriptome data of $P$. lobata, a qRT-PCR was carried out on the PIKOREAL 96 PCR System (Thermo Scientific, Waltham, MA, USA) with the Novostart SYBR qPCR SuperMix Plus kit (Novoprotein, Shanghai, China). Primer pairs were designed to amplify the actin gene and nine unigenes involved in isoflavonoid biosynthesis using Primer Premier (v5.0) (Supplementary Table S3). All reactions were prepared in a final volume of $10 \mu \mathrm{l}$ (containing $1 \mu \mathrm{l}$ of cDNA, $1 \mu \mathrm{l}$ of each specific primer, $5 \mu \mathrm{L}$ of $2 \times$ SYBR Green mixture, and $2 \mu \mathrm{L}$ of RNase-free water). The qRT-PCR was run under these conditions: 1 min at $95^{\circ} \mathrm{C}, 40$ cycles of $95^{\circ} \mathrm{C}$ for $20 \mathrm{~s}$, and $60^{\circ} \mathrm{C}$ for $1 \mathrm{~min}$. Relative expression levels were normalized to those of actin (CL10129.Contig5) mRNA, by applying the $2^{-\Delta \Delta C t}$ method(Livak \& Schmittgen 2001). Every target gene was run in three biological replicates.

PeerJ reviewing PDF | (2020:07:51383:4:0:NEW 27 Dec 2020) 
153

154

155

156

157

158

159

160

161

162

163

164

165

166

167

168

169

170

171

172

173

174

175

176

177

178

179

180

181

182

183

\section{Determination of the content of isoflavonoid components in different $P$. lobata tissues}

The quantitative analysis of isoflavonoid metabolites of different $P$. lobata tissues was done using HPLCMS/MS. The resulting mass spectra indicated the presence of six isoflavonoid metabolites in $P$. lobata roots (puerarin, $2.649 \mathrm{mg} / \mathrm{g}$; daidzin, $1.105 \mathrm{mg} / \mathrm{g}$; glycitin, $0.196 \mathrm{mg} / \mathrm{g}$; genistin, $0.0834 \mathrm{mg} / \mathrm{g}$; daidzein, $0.052 \mathrm{mg} / \mathrm{g}$; formononetin, $0.004 \mathrm{mg} / \mathrm{g}$, for which $\mathrm{mg} / \mathrm{g}$ is the fresh weight of roots), three metabolites in leaves (puerarin, $0.011 \mathrm{mg} / \mathrm{g}$; genistin, $0.002 \mathrm{mg} / \mathrm{g}$; genistein, $0.001 \mathrm{mg} / \mathrm{g}$, for which $\mathrm{mg} / \mathrm{g}$ is the fresh weight of leaves), and four metabolites in stems (puerarin, $0.005 \mathrm{mg} / \mathrm{g}$; genistin, $0.005 \mathrm{mg} / \mathrm{g}$; genistein, $0.001 \mathrm{mg} / \mathrm{g}$; daidzein, 0.001 $\mathrm{mg} / \mathrm{g}$, for which $\mathrm{mg} / \mathrm{g}$ is the fresh weight of stems), according to their respective retention time, molecular ion peak, and ESI-MS data (Fig. 1, Supplementary Fig. 2). The content of isoflavonoid components was the highest in the roots (at $4.089 \mathrm{mg} / \mathrm{g}$ ), with substantially lower amounts in the leaves (at $0.014 \mathrm{mg} / \mathrm{g}$ ) and stems $(0.013 \mathrm{mg} / \mathrm{g})$; in this respect, the difference between roots and other tissue types was statistically significant.

\section{Illumina sequencing and de novo assembly of the $P$. lobata transcriptome}

The RNA-Seq analysis of $P$. lobate roots, leaves, and stems generated $31.63 \mathrm{~Gb}$ of high-quality reads, characterized by a Q30-values $\geq 89.25 \%$ (Supplementary Table S4). After assembling these high-quality reads per tissue type, their full-length transcripts were sequentially reconstructed. After selecting the longest transcript of each, a total of 140,905 unigenes were obtained (using software TGICL). These had a mean length of $1083 \mathrm{bp}$ and an N50 value of $1883 \mathrm{bp}$; moreover, 38.47\% (54,209) and 26.13\% (36,821) of these unigenes exceeded 1000 bp and 1500 bp in length, respectively (Supplementary Fig. 3; Table S5).

\section{Functional annotation and expression overview of unigenes}

Of the 140,905 unigenes, we were able to map $70.60 \%, 58.04 \%, 55.30 \%, 55.03 \%, 53.67 \%, 49.62 \%$, and $47.29 \%$ of them to the NR, NT, GO, KOG, KEGG, SwissProt, and Pfam databases, respectively. Thus, a total of 109,687 (77.84\%) unigenes were mapped to at least one public database, while $26.61 \%$ unigenes $(37,498)$ were co-annotated in all seven databases (Table 1). A species distribution analysis showed that $P$. lobata unigenes had the highest homology to Glycine soja sequences (29,597 unigenes; 29.75\%), followed by Quercus suber (18,164 unigenes; 18.26\%) and Glycine max (16,822 unigenes; 16.91\%) (Supplementary Fig. 4).

The GO database mapped 77,920 unigenes to "biological process", "cellular component" and "molecular function", with every unigene categorized to at least one GO term. Additionally, the abundant genes could be divided into "cellular process" (35,585 unigenes [45.67\%]) and "metabolic process" (31,031 unigenes [39.82\%]) terms under "biological process"; under the "molecular function", the "binding" (42,267 unigenes 
184

185

186

187

188

189

190

191

192

193

194

195

196

197

198

199

200

201

202

203

204

205

206

207

208

209

210

211

212

213

214

215

216

[54.24\%]), and "catalytic activity" (40,388 unigenes [51.83\%]) were the most abundant GO terms characterizing the unigenes (Supplementary Fig. 5).

Unigenes with fragments per kilobase of transcript per million (FPKM) values were counted in different tissues of $P$. lobata plants. We found 42,591, 49,524, and 48,806 unigenes in the leaf, stem, and root tissues with an FPKM $\leq 1$, which corresponded to low-level expression; correspondingly, there were 29,780, 43,555, and 34,229 unigenes with an FPKM =1-10, indicative of medium-level expression, and 9999, 10,860, and 10,063 unigenes with an FPKM $\geq 10$ that exhibited high-level expression (Fig. 2a). Overall, the expression level was greatest in stems compared with the roots and leaves (Fig. 2b).

\section{Identification of structural genes involved in isoflavonoid biosynthesis}

To identify the most significant biological pathways in $P$. lobata plants, functional annotations using the KEGG database mapped 75,628 unigenes to 19 subcategories (137 pathways). Of the latter, 11 were related to metabolism and most unigenes were assigned to "carbohydrate metabolism" (7174 unigenes), followed by “amino acid metabolism" (3888 unigenes), "lipid metabolism” (3301 unigenes), “energy metabolism” (2574 unigenes), "biosynthesis of other secondary metabolites" (2567 unigenes) (Fig. 3A; Supplementary Fig. 6, Table S6, S7 and S8). Within the biosynthesis of the other set of secondary metabolites, the largest number of unigenes were found involved in the "phenylpropanoid biosynthesis" (1590 unigenes), "flavonoid biosynthesis", "isoquinoline alkaloid biosynthesis", "tropane, piperidine, and pyridine alkaloid biosynthesis", and "isoflavonoid biosynthesis" pathways (Fig. 3B; Supplementary S7 and S8).

Additionally, to enhance our understanding of isoflavonoid biosynthesis, we annotated 1590 and 314 unigenes involved in the phenylpropanoid (ko00940) and flavonoid biosynthesis (ko00941) pathways, respectively. A total of 94 unigenes that encoded key enzymes were identified under "isoflavonoid biosynthesis" (ko00900): these were phenylalanine ammonia-lyase (PAL; 8 unigenes), 4-coumarate-CoA ligase (4CL; 17 unigenes), trans-cinnamate 4-monooxygenase (C4H; 9 unigenes), chalcone synthase (CHS; 7 unigenes), chalcone isomerase (CHI; 19 unigenes), 2-hydroxyisoflavanone synthase (IFS2; 11 unigenes), flavonoid 6-hydroxylase (F6H; 1 unigenes), isoflavone 4'-O-methyltransferase (IOMT; 4 unigenes), 2hydroxyisoflavanone dehydratase (HIDH; 11 unigenes) and isoflavone 7-O-glucosyltransferase (IF7GT; 7 unigenes) (Table 2; Supplementary Table S9). A heatmap was used to visualize patterns in the expression levels of these enzyme-encoding unigenes involved in isoflavonoid biosynthesis pathways (Fig. 4). Most of the unigenes encoding PAL, C4H, CHS, F6H, and IF7GT were expressed at higher levels in roots, while some unigenes encoding IOMT and HIDH were expressed more in leaves. Unigenes encoding 4CL, CHI, and IFS2 were all highly expressed in stems (Fig. 4).

In our assembled transcriptome for $P$. lobata, a total of 298 unigenes encoding the UDP glycosyltransferases

Peer) reviewing PDF | (2020:07:51383:4:0:NEW 27 Dec 2020) 
217 (UGTs) were identified, which included 137 full-length open reading frames (ORFs) of the unigenes 218 (Supplementary Table S10). Furthermore, seven unigenes (CL4436-1, CL4436-4, CL4436-5, CL4436-6, 219 CL4436-8, CL4436-9, Un-54862) were predicted to encode the isoflavone 7-O-glucosyltransferase (IF7GT) 220 known to be involved in isoflavonoid biosynthesis, whose expression among tissues was greatest in the roots. 221 The amino acid sequence of IF7GT in P. lobatarevealed a highly conserved region within the C-terminal 222 domain — known as a plant secondary product glycosyltransferase (GT) consensus sequence (called PSPG) 223 involved in secondary metabolism when compared with the other plant UGTs of G. max (NP_001304440.2; 224 93.53\%), G. soja (XP_028207064.1; 93.28\%), and P. montana var.lobata (AMQ26113.1; 90.55\%).

225

The secondary structure of IF7GT contained $12 \beta$-sheets and $14 \alpha$-helices, with the regions of $\beta$-sheet 10 to $\alpha$ helix 14 potentially involved in binding the protein to the UDP moiety of sugar (depicted by the black box in Fig. 5).

\section{Validation of RNA-Seq data using qRT-PCR}

To verify whether the differential gene expression found among different $P$. lobata tissues by the FPKM analysis was accurate, nine unigenes participating in isoflavonoid biosynthesis were chosen for a qRT-PCR analysis. The expression levels of the six unigenes including C4H (CL2444.Contig2), 4CL (CL1520.Contig3), CHS (CL3338.Contig1), IFS2 (CL2625.Contig2), HIDH (CL10538.Contig1), and IF7GT (Unigene54862) were all consistent between the qRT-PCR analysis and RNA-Seq data. Furthermore, the qRT-PCR results corroborated that the expression levels of these selected genes were highest in root tissue (Fig. 6).

\section{Identification of transcription factors (TFs) involved in the biosynthesis of isoflavonoid and other secondary metabolites}

The mechanism regulating isoflavonoid metabolic processes is considered to operate primarily at the transcription level, so TF families likely play key roles in this by specifically binding to cis-regulatory elements in the promoter regions of involved genes. A total of 2985 unigenes were identified as putative TFs in the P. lobata transcriptome when using Hmmsearch (v3.0) to search the plant transcription factor database (PlantTFDB). These included 905 and 474 unigenes up-regulated in roots compared with stems and leaves, respectively (Table 3; Supplementary Table S11). Most of these TF-encoding unigenes were annotated to MYB (375 unigenes), bHLH (221 unigenes), WRKY (208 unigenes), AP2-EREBP (196 unigenes), NAC (170 unigenes), $\mathrm{C} 3 \mathrm{H}$ (158 unigenes), and $\mathrm{C} 2 \mathrm{H} 2$ (156 unigenes) families. Furthermore, we found that the unigenes encoding the MYB (sixunigenes), TCP (three unigenes), Trihelix (two unigenes), as well as BSD, C2H2, GeBP, WRKY, mTERF, and zf-HD (one unigene each) TFs were involved in phenylpropanoid biosynthesis, 
249

250

251

252

253

254

255

256

257

258

259

260

261

262

263

264

265

266

267

268

269

270

271

272

273

274

275

276

277

278

279

280

providing the precursors for isoflavonoid metabolism. We also uncovered a total of 14 unigenes encoding several TFs (AP2-EREBP, MYB, RWP-RK, and zf-HD) involved in "betalain biosynthesis", "carotenoid biosynthesis", and "tropane, piperidine and pyridine alkaloid" secondary metabolites.

\section{Identification of DEGs and analysis of potential TFs related to isoflavonoid biosynthesis}

Among the unigenes identified in three different tissues of P. lobata, 18,170 were expressed specifically in roots, while 62,297 were expressed in all three tissues (Fig. 7A). DEGs were detected among tissue types according to the FPKM values of unigenes (Fig. 7B). A total of 38,751 DEGs between the root and leaf transcriptome were detected, of which 17,064 were down-regulated and 21,687 up-regulated in roots compared with leaves. Comparing the unigene expression levels between roots and stems revealed 36,155 DEGs, of which 21,884 were down-regulated and 14,271 were up-regulated in roots compared with stems. Far less DEGs $(19,790)$ were identified when roots were compared to both leaves and stems, with 9440 of these downregulated and 10,350 up-regulated.

In addition to the structural unigenes, 19,790 co-expression DEGs were further analyzed to look for other regulatory unigenes, such as TFs, that were potentially related to the isoflavonoid biosynthesis. We found 798 co-expression DEGs were predicted to be TF families and it is hypothesized that isoflavonoid-related TFs should have highly correlated expression levels with those of structural genes. Thus, the expression levels of 798 TFs were calculated using Pearson's correlation coefficients ( $r)(r>0.8$ or $r<-0.8)$ with those of PAL (CL518.Contig3), C4H (CL2444.Contig2), 4CL (CL1520.Contig3), CHS (CL3338.Contig1), CHI (Unigene9945), F6H (Unigene72602), IFS2 (CL2625.Contig2), HIDH (CL10538.Contig1), and IF7GT (Unigene54862). In total, $608 \mathrm{TFs}$ that were coexpressed with at least 1 of the 9 structural unigenes involved in isoflavonoid biosynthesis were screened (Supplementary Table S12). Among them, we also identified 426, 16, $45,17,8,15,31,45$ and 5 TFs were negatively or positively correlated with the $9,8,7,6,5,4,3,2$, and 1 structural unigene, respectively (Supplementary Table S12). The 608 TFs were classified into 49 families, and most of the unigenes were enriched to the MYB, bHLH, WRKY, AP2-EREBP and C2H2 TF families.

\section{Analysis of root-specific expression unigenes}

A total of 10,350 up-regulated DEGs that exhibited root-specific expression, with values of $\log 2 \mathrm{FC}>1$, were mapped to 54 subcategories in the three functional categories of GO database. Within the biological processes and molecular function categories, most genes were significantly enriched under "cellular process", "metabolic process", and "catalytic activity", and "binding"categories (Fig. 8A; Supplementary Table S13). Within the KEGG database, these DEGs having root-specific expression were further annotated to 134 pathways. Among 
281 the latter, most DEGs were significantly enriched in "flavonoid biosynthesis" (Rich ratio $=0.283$ ),

282 "isoflavonoid biosynthesis" (Rich ratio = 0.259), "stilbenoid, diarylheptanoid and gingerol biosynthesis" (Rich 283 ratio $=0.241)$, "phenylpropanoid biosynthesis" $($ Rich ratio $=0.181)$, and "lysine biosynthesis" $($ Rich ratio $=$ 284 0.179) (Fig. 8B).

285

286

The roots of $P$. lobata, which are the main plant part prescribed in traditional Chinese medicines to treat various diseases, produce predominantly isoflavone $\mathrm{O}$ - and $\mathrm{C}$-glucosides. The three tissues from which the concentrations of isoflavonoids were detected using HPLC-MS/MS were also analyzed for deep transcriptomic data on the BGISEQ-500 system. Isoflavonoids clearly accumulate to a much higher level in roots than either leaves or stems, and the content of puerarin exceeds that of daidzin, glycitin, genistin, genistein, daidzein, and formononetin (Fig.1). Further, the concentration of puerarin in roots not only differs from leaves and stems, but it also varies among different root areas of the $P$. lobata plant(Du et al. 2010). This unequal distribution may arise from essential enzymes for the accumulation of puerarin being actively expressed in the roots with low or no expression in other plant tissue types. Thus, it is important to investigate these DEGs since this could advance the functional research of $P$. lobata plants with high contents of special isoflavonoids, to expand their usage. The analysis of the transcriptome dataset in our study revealed 140,905 unigenes, 109,687 of which were annotated, leaving $22.16 \%$ non-annotated, probably due to insufficient data on plant transcriptomes and genomes currently in the NCBI database. Nonetheless, the Nr annotations showed that 29,597 (29.75\%) of the P. lobata unigenes had higher homology to the G.soja protein database, this having a higher similarity of sequences than with either Q.suber or G.max. This result suggested P. lobata is more closely related to the $G$.soja and so the latter's genome could be taken as a reference for future functional genomics research in $P$. lobata.

We performed RNA-Seq analyses of roots, stems, and leaves of $P$. lobata to facilitate the dissection of differentially expressed genes involved in the tissue-specific biosynthesis of isoflavonoid. This approach has been applied to identify the novel genes involved in the secondary metabolism pathways and analysis of the molecular mechanism of isoflavonoid biosynthesis(Dastmalchi \& Dhaubhadel 2015; Suntichaikamolkul et al. 2019; Wang et al. 2018). Comparative analysis of $P$. lobata roots and leaves and stems expression profiles exhibited 10,350 root-specific DEGs, and that these DEGs were significantly enriched in flavonoid biosynthesis and isoflavonoid biosynthesis (Fig.8B). These root-specific DEGs may explain the molecular mechanism of the medicinal value of $P$. lobata roots.

Isoflavonoids are the major bioactive components in $P$. lobata, although the relationship between the expression of structural genes in isoflavonoid biosynthesis and the accumulation of isoflavonoids in P. lobata is unclear. In the transcriptome dataset of $P$. lobata, we identified 94 putative structural unigenes that were 
314

involved either upstream or downstream of the isoflavonoid biosynthesis pathway (Fig.4). Our HPLC-MS/MS analysis confirmed that the content of isoflavonoids in P. lobata was the highest in roots compared with other tissues. Higher expression levels of some unigenes encoding F6H, HIDH, and IF7GT in roots compared with leaves and stems were consistent with the fact higher isoflavonoid accumulation occurred in $P$. lobata roots (Fig. 1, Fig. 4, Fig. 6). Taken together, these results suggest overexpression of these genes could increase the accumulation of isoflavonoids in P. lobata plants. Previous studies have shown that overexpression of the HIDH gene increased the productivity of daidzein and genistein in Lotus japonicas roots(Shimamura et al. 2007), and that the expression level of a novel isoflavone 7-O-glucosyltransferase (PIUGT1) was correlated with the accumulation pattern of isoflavone glycosides in P. lobata roots(Li et al. 2014).

In plants, UDP glycosyltransferases (UGTs) utilize UDP-activated sugars as donors and transfer their sugar moiety to acceptor molecules, which play central roles in regulating isoflavonoid activities and detecting the diversity of their structures( $\mathrm{Li}$ et al. 2007; Noguchi et al. 2007). Within the P. lobata transcriptome, seven fulllength IF7GT candidates were selected that had their highest expression levels in roots. Multiple sequence alignment of the IF7GT amino acids revealed a highly conserved region in the PSPG-box (Fig. 5), which is similar to the original GT consensus sequence, with a short stretch of $\sim 40$ amino acids at the C-terminal part of the protein(Bairoch 1992; Vogt \& Jones 2000). GTs are generally involved in plant secondary metabolism, but this depends on the inversion of the anomeric sugar from an $\alpha$ linkage in UDP-glucose to the $\beta$ configuration in resultant glycoside(Dmitri \& Yu 1999). Two highly conserved sequences (WAPQA and HCGWNS) of IF7GT were located at $\beta 10, \alpha 12, \beta 11$, and $\alpha 13$, and they may correspond to the active site of GTs in P. lobata. Thus, our findings can assist further investigation into the structures of IF7GTs and the regulation of isoflavonoid activities.

In addition to the structural unigenes, we also analyzed TFs regulatory unigenes. TFs are sequence-specific DNA-binding proteins that can either induce or repress the accumulation of specific metabolites, quite effectively, by regulating the expression of target genes(Butelli et al. 2008; Cutanda-Perez et al. 2009; Shelton et al. 2012). For example, overexpression of the LjMYB14 gene can enhance the expression of genes that encode PAL, C4H, and 4CL enzymes involved in the isoflavonoid biosynthesis of Lotus japonicas(Shelton et al. 2012). In soybean plant, over-expression of GmMYB176 by activating CHS8 gene promoter affected isoflavone biosynthesis(Yi et al. 2010). Conversely, over-expression of GmMYB39 in soybean was able to reduce the expression levels of PAL, C4H, CHS, and 4CL, yet it slightly increased that of IFS(Liu et al. 2013). Here, we found that the expression level of MYB39 (XM_003541813.2) was positively correlated with that of PAL, C4H, 4CL, CHS, CHI, F6H, IFS2, HIDH, and IF7GT in P. lobata. Additionally, we also identified TFs of bHLH, WRKY, AP2-EREBP, C2H2, NAC,G2-like and MADS that were potentially involved in isoflavonoid biosynthesis by regulating multiple structural unigenes (Supplementary Table S12). Knowledge of these TFs existence could foster functional studies of transcriptional activation of key enzymes related to isoflavonoid synthesis in P. lobata. 


\section{CONCLUSIONS}

351 Overall, deep transcriptome analysis of root, leaf, and stem tissues of P. lobata plants were done for 352 identifying candidate genes involved in this plant's biosynthesis of isoflavonoids. The results of this RNA-Seq 353 analysis will facilitate further study of the molecular mechanisms of isoflavonoid biosynthesis in $P$. lobata and 354 should allow us to analyze the functional genomics other pathways that are currently less well understood.

\section{ACKNOWLEDGEMENTS}

357 We thank the Beijing Genomics Institute for assistance with the experiments.

358

359

360

361

362

363

364

365

366

367

368

369

370

371

372

\section{ADDITIONAL INFORMATION AND DECLARATIONS}

\section{Author Contributions}

- Chenkai Wang performed the experiments, analyzed the data, wrote the paper and prepared figures and /or tables.

-Nenggui Xu conceived and designed the experiments, and analyzed the data.

-Shuai Cui conceived and designed the experiments, and performed the experiments.

\section{Competing Interests}

The authors declare that they have no competing interests.

\section{Data Availability}

The sequencing data of $P$. lobata have been deposited in National Center for Biotechology Information (NCBI) Sequence Read Archive (SRA) database under the accession number PRJNA630835 or SRP261192 (https://www.ncbi.nlm.nih.gov/Traces/study/?acc=PRJNA630835). 


\section{REFERENCES}

374

Bairoch A. 1992. PROSITE: a dictionary of sites and patterns in proteins. Nucleic Acids Res 20 Suppl:2013-2018. 10.1093/nar/20.suppl.2013

Bertoni M, Kiefer F, Biasini M, Bordoli L, and Schwede T. 2017. Modeling protein quaternary structure of homoand hetero-oligomers beyond binary interactions by homology. 7:10480. 10.1038/s41598-017-09654-8

Butelli E, Titta L, Giorgio M, Mock HP, Matros A, Peterek S, Schijlen EG, Hall RD, Bovy AG, Luo J, and Martin C. 2008. Enrichment of tomato fruit with health-promoting anthocyanins by expression of select transcription factors. Nat Biotechnol 26:1301-1308. 10.1038/nbt.1506

Carai MA, Agabio R, Bombardelli E, Bourov I, Gessa GL, Lobina C, Morazzoni P, Pani M, Reali R, Vacca G, and Colombo G. 2000. Potential use of medicinal plants in the treatment of alcoholism. Fitoterapia 71 Suppl 1:S38-42. 10.1016/s0367-326x(00)00178-7

Chang Y, Wang M, Li J, and Lu S. 2019. Transcriptomic analysis reveals potential genes involved in tanshinone biosynthesis in Salvia miltiorrhiza. 9:14929. 10.1038/s41598-019-51535-9

Chen X, Yu J, and Shi J. 2018. Management of Diabetes Mellitus with Puerarin, a Natural Isoflavone From Pueraria lobata. Am J Chin Med 46:1771-1789. 10.1142/s0192415x18500891

Chen Y, Chen Y, Shi C, Huang Z, Yong Z, Li S, Yan L, Jia Y, Chang Y, and Zhuo L. 2017. SOAPnuke: a MapReduce acceleration-supported software for integrated quality control and preprocessing of high-throughput sequencing data. Gigascience:1.

Conesa A, and Götz S. 2008. Blast2GO: A comprehensive suite for functional analysis in plant genomics. Int J Plant Genomics 2008:619832. 10.1155/2008/619832

Cutanda-Perez MC, Ageorges A, Gomez C, Vialet S, Terrier N, Romieu C, and Torregrosa L. 2009. Ectopic expression of VImybA1 in grapevine activates a narrow set of genes involved in anthocyanin synthesis and transport. Plant Mol Biol 69:633-648. 10.1007/s11103-008-9446-x

Dastmalchi M, and Dhaubhadel S. 2015. Proteomic insights into synthesis of isoflavonoids in soybean seeds. Proteomics 15:1646-1657. 10.1002/pmic.201400444

Deavours BE, and Dixon RA. 2005. Metabolic engineering of isoflavonoid biosynthesis in alfalfa. Plant Physiol 138:2245-2259. 10.1104/pp.105.062539

Dembélé D, and Kastner P. 2014. Fold change rank ordering statistics: a new method for detecting differentially 
Dmitri K, and Yu RK. 1999. Conserved domains of glycosyltransferases. Glycobiology:10.

403

404

405

406

407

408

409

410

411

412

413

414

415

416

417

418

419

420

421

422

423

424

425

426

427

428

429

Du G, Zhao HY, Zhang QW, Li GH, Yang FQ, Wang Y, Li YC, and Wang YT. 2010. A rapid method for simultaneous determination of 14 phenolic compounds in Radix Puerariae using microwave-assisted extraction and ultra high performance liquid chromatography coupled with diode array detection and time-of-flight mass spectrometry. J Chromatogr A 1217:705-714. 10.1016/j.chroma.2009.12.017

Garg R, and Jain M. 2013. RNA-Seq for Transcriptome Analysis in Non-model Plants. Methods in Molecular Biology 1069:43-58.

Grabherr MG, Haas BJ, Yassour M, Levin JZ, and others. 2013. Trinity: reconstructing a full-length transcriptome without a genome from RNA-Seq data. Nature Biotechnology 29:644.

Han R, Takahashi H, Nakamura M, Yoshimoto N, Suzuki H, Shibata D, Yamazaki M, and Saito K. 2015. Transcriptomic landscape of Pueraria lobata demonstrates potential for phytochemical study. Front Plant Sci 6:426. 10.3389/fpls.2015.00426

He M, Yao Y, Li Y, Yang M, Li Y, and Wu B. 2019. Comprehensive transcriptome analysis reveals genes potentially involved in isoflavone biosynthesis in Pueraria thomsonii Benth. 14:e0217593. 10.1371/journal.pone.0217593

He X, Blount JW, Ge S, Tang Y, and Dixon RA. 2011. A genomic approach to isoflavone biosynthesis in kudzu (Pueraria lobata). Planta 233:843-855. 10.1007/s00425-010-1344-1

Jin SE, Son YK, Min BS, Jung HA, and Choi JS. 2012. Anti-inflammatory and antioxidant activities of constituents isolated from Pueraria lobata roots. Arch Pharm Res 35:823-837. 10.1007/s12272-012-0508-x

Kim JA, Roy NS, Lee IH, Choi AY, Choi BS, Yu YS, Park NI, Park KC, Kim S, Yang HS, and Choi IY. 2019. Genome-wide transcriptome profiling of the medicinal plant Zanthoxylum planispinum using a single-molecule direct RNA sequencing approach. Genomics 111:973-979. 10.1016/j.ygeno.2018.06.004

Kim KI, and van de Wiel MA. 2008. Effects of dependence in high-dimensional multiple testing problems. Bmc Bioinformatics 9:114. 10.1186/1471-2105-9-114

Langmead B, and Salzberg SL. 2012. Fast gapped-read alignment with Bowtie 2. Nat Methods 9:357-359. 10.1038/nmeth.1923

Li B, and Dewey CN. 2011. RSEM: accurate transcript quantification from RNA-Seq data with or without a reference genome. Bmc Bioinformatics 12:323.

Peer) reviewing PDF | (2020:07:51383:4:0:NEW 27 Dec 2020) 
430

431

432

Li J, Li C, Gou J, and Zhang Y. 2016. Molecular Cloning and Functional Characterization of a Novel Isoflavone 3'-Omethyltransferase from Pueraria lobata. Front Plant Sci 7:793. 10.3389/fpls.2016.00793

Li J, Li Z, Li C, Gou J, and Zhang Y. 2014. Molecular cloning and characterization of an isoflavone 7-Oglucosyltransferase fromPueraria lobata. Plant Cell Reports 33:1173-1185.

Li L, Modolo LV, Escamilla-Trevino LL, Achnine L, Dixon RA, and Wang X. 2007. Crystal Structure of Medicago truncatula UGT85H2 - Insights into the Structural Basis of a Multifunctional (Iso)flavonoid Glycosyltransferase. Journal of Molecular Biology 370:0-963.

Liu X, Yuan L, Xu L, Xu Z, Huang Y, He X, Ma H, Yi J, and Zhang D. 2013. Over-expression of GmMYB39 leads to an inhibition of the isoflavonoid biosynthesis in soybean (Glycine max. L). Plant Biotechnology Reports 7:445455. $10.1007 / \mathrm{s} 11816-013-0283-2$

Livak KJ, and Schmittgen TD. 2001. Analysis of relative gene expression data using real-time quantitative PCR and the 2(-Delta Delta C(T)) Method. Methods 25:402-408. 10.1006/meth.2001.1262

Meezan E, Meezan EM, Jones K, Moore R, Barnes S, and Prasain JK. 2005. Contrasting effects of puerarin and daidzin on glucose homeostasis in mice. J Agric Food Chem 53:8760-8767. 10.1021/jf058105e

Mistry J, Finn RD, Eddy SR, Bateman A, and Punta M. 2013. Challenges in homology search: HMMER3 and convergent evolution of coiled-coil regions. Nucleic Acids Res 41:e121. 10.1093/nar/gkt263

Miyazawa M, Sakano K, Nakamura S, and Kosaka H. 2001. Antimutagenic activity of isoflavone from Pueraria lobata. J Agric Food Chem 49:336-341. 10.1021/jf000255w

Noguchi A, Saito A, Homma Y, Nakao M, Sasaki N, Nishino T, Takahashi S, and Nakayama T. 2007. A UDPglucose:isoflavone 7-0-glucosyltransferase from the roots of soybean (glycine max) seedlings. Purification, gene cloning, phylogenetics, and an implication for an alternative strategy of enzyme catalysis. $J$ Biol Chem 282:23581-23590. 10.1074/jbc.M702651200

Ohshima Y, Okuyama T, Takahashi K, Takizawa T, and Shibata S. 1988. Isolation and high performance liquid chromatography (HPLC) of isoflavonoids from the Pueraria root. Planta Med 54:250-254. 10.1055/s-2006962420

Ozsolak F, and Milos PM. 2011. RNA sequencing: advances, challenges and opportunities. Nat Rev Genet 12:87-98. $10.1038 / \mathrm{nrg} 2934$

Pan ZJ, Yan GL, Wang LP, Xu XX, and Pan L. 2011. [Effects of puerarin on blood pressure, blood lipid and renal structure of cold-induced hypertensive mice]. Zhongguo Ying Yong Sheng Li Xue Za Zhi 27:436-438.

Peer) reviewing PDF | (2020:07:51383:4:0:NEW 27 Dec 2020) 
459

460

461

462

463

464

465

466

467

468

469

470

471

472

473

474

475

476

477

478

479

480

481

482

483

484 485

486

487

488
Pertea G, Huang X, Liang F, Antonescu V, Sultana R, Karamycheva S, Lee Y, White J, Cheung F, Parvizi B, Tsai J, and Quackenbush J. 2003. TIGR Gene Indices clustering tools (TGICL): a software system for fast clustering of large EST datasets. Bioinformatics 19:651-652. 10.1093/bioinformatics/btg034

Rice P, Longden I, and Bleasby A. 2000. EMBOSS: The European molecular biology open software suite. Trends in Genetics 16:276-277.

Robert X, and Gouet P. 2014. Deciphering key features in protein structures with the new ENDscript server. Nucleic Acids Res 42:W320-324. 10.1093/nar/gku316

Shelton D, Stranne M, Mikkelsen L, Pakseresht N, Welham T, Hiraka H, Tabata S, Sato S, Paquette S, Wang TL, Martin C, and Bailey P. 2012. Transcription factors of Lotus: regulation of isoflavonoid biosynthesis requires coordinated changes in transcription factor activity. Plant Physiol 159:531-547. $10.1104 /$ pp.112.194753

Shimamura M, Akashi T, Sakurai N, Suzuki H, Saito K, Shibata D, Ayabe S-i, and Aoki aT. $2007.2-$ Hydroxyisoflavanone Dehydratase is a Critical Determinant of Isoflavone Productivity in Hairy Root Cultures of Lotus japonicus. Plant \& Cell Physiology 48:1652-1657.

Soltani Howyzeh M, Sadat Noori SA, Shariati JV, and Amiripour M. 2018. Comparative transcriptome analysis to identify putative genes involved in thymol biosynthesis pathway in medicinal plant Trachyspermum ammi L. Sci Rep 8:13405. 10.1038/s41598-018-31618-9

Suntichaikamolkul N, Tantisuwanichkul K, Prombutara P, Kobtrakul K, Zumsteg J, Wannachart S, Schaller H, Yamazaki M, Saito K, De-Eknamkul W, Vimolmangkang S, and Sirikantaramas S. 2019. Transcriptome analysis of Pueraria candollei var. mirifica for gene discovery in the biosyntheses of isoflavones and miroestrol. 19:581. 10.1186/s12870-019-2205-0

Vashisht I, Pal T, Sood H, and Chauhan RS. 2016. Comparative transcriptome analysis in different tissues of a medicinal herb, Picrorhiza kurroa pinpoints transcription factors regulating picrosides biosynthesis. Mol Biol Rep 43:1395-1409. 10.1007/s11033-016-4073-0

Vogt T. 2010. Phenylpropanoid biosynthesis. Mol Plant 3:2-20. 10.1093/mp/ssp106

Vogt T, and Jones P. 2000. Glycosyltransferases in plant natural product synthesis: characterization of a supergene family. Trends Plant Sci 5:380-386. 10.1016/s1360-1385(00)01720-9

Wang C, Zhu J, Liu M, Yang Q, Wu J, and Li Z. 2018. De novo sequencing and transcriptome assembly of Arisaema heterophyllum Blume and identification of genes involved in isoflavonoid biosynthesis. Sci Rep 8:17643. $10.1038 / \mathrm{s} 41598-018-35664-1$

Peer) reviewing PDF | (2020:07:51383:4:0:NEW 27 Dec 2020) 
489

490

491

492

493

494

495

496

497

498

499

500

501

502

503

504

505

506

507

508
Wang X, Li C, Zhou C, Li J, and Zhang Y. 2017. Molecular characterization of the C-glucosylation for puerarin biosynthesis in Pueraria lobata. Plant J 90:535-546. 10.1111/tpj.13510

Wang Z, Gerstein M, and Snyder M. 2009. RNA-Seq: a revolutionary tool for transcriptomics. Nat Rev Genet 10:5763. $10.1038 / \mathrm{nrg} 2484$

Wong KH, Li GQ, Li KM, Razmovski-Naumovski V, and Chan K. 2011. Kudzu root: traditional uses and potential medicinal benefits in diabetes and cardiovascular diseases. J Ethnopharmacol 134:584-607. 10.1016/j.jep.2011.02.001

Yang Y, Jiang XT, and Zhang T. 2014. Evaluation of a hybrid approach using UBLAST and BLASTX for metagenomic sequences annotation of specific functional genes. Plos One 9:e110947. 10.1371/journal.pone.0110947

Yi J, Derynck MR, Li X, Telmer P, Marsolais F, and Dhaubhadel S. 2010. A single repeat MYB transcription factor, GmMYB176, regulates CHS8 gene expression and affects isoflavonoid biosynthesis in soybean. Plant J. Plant Journal 62:1019-1034.

Zhang H, He L, and Cai L. 2018. Transcriptome Sequencing: RNA-Seq. Methods Mol Biol 1754:15-27. 10.1007/9781-4939-7717-8_2

Zhu FY, Chen MX, Ye NH, Qiao WM, Gao B, Law WK, Tian Y, Zhang D, Zhang D, Liu TY, Hu QJ, Cao YY, Su ZZ, Zhang J, and Liu YG. 2018. Comparative performance of the BGISEQ-500 and Illumina HiSeq4000 sequencing platforms for transcriptome analysis in plants. Plant Methods 14:69. 10.1186/s13007-018-0337-0 
Figure 1

Representative chromatograms of the isoflavonoid analyzed in Pueraria lobata three tissues

(a) standard sample solution; (b), (c) and (d) are respectively the root, leaf, and stem tissues. On the $\mathrm{x}$-axis is the retention time; on the $\mathrm{y}$-axis are the intensity values. 


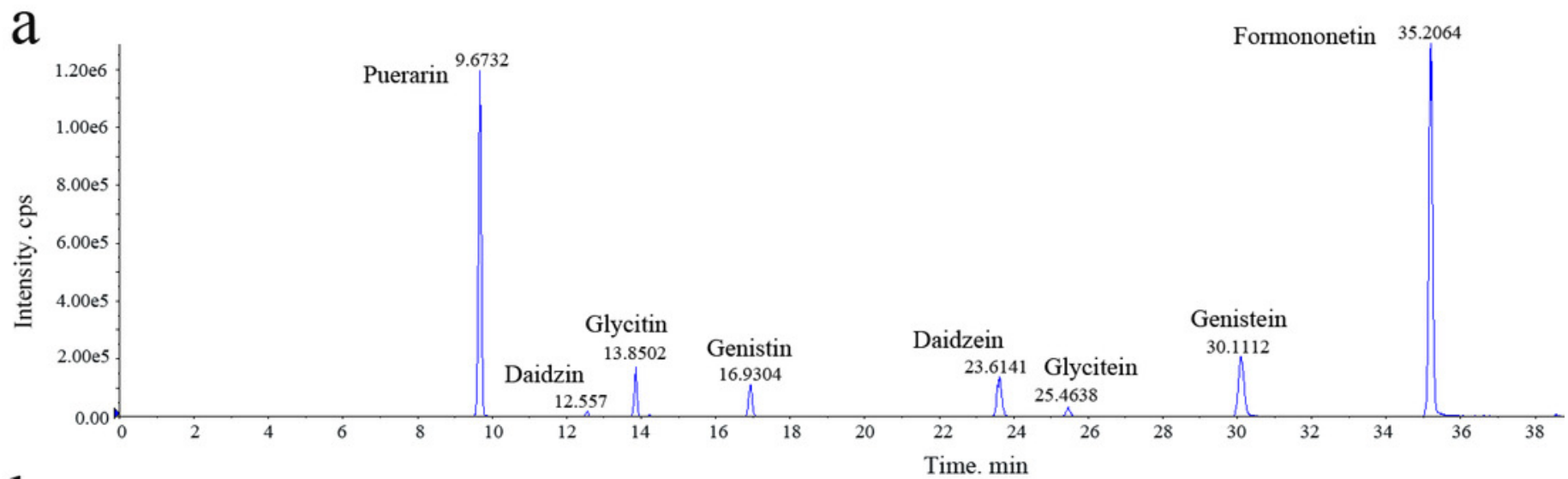

b
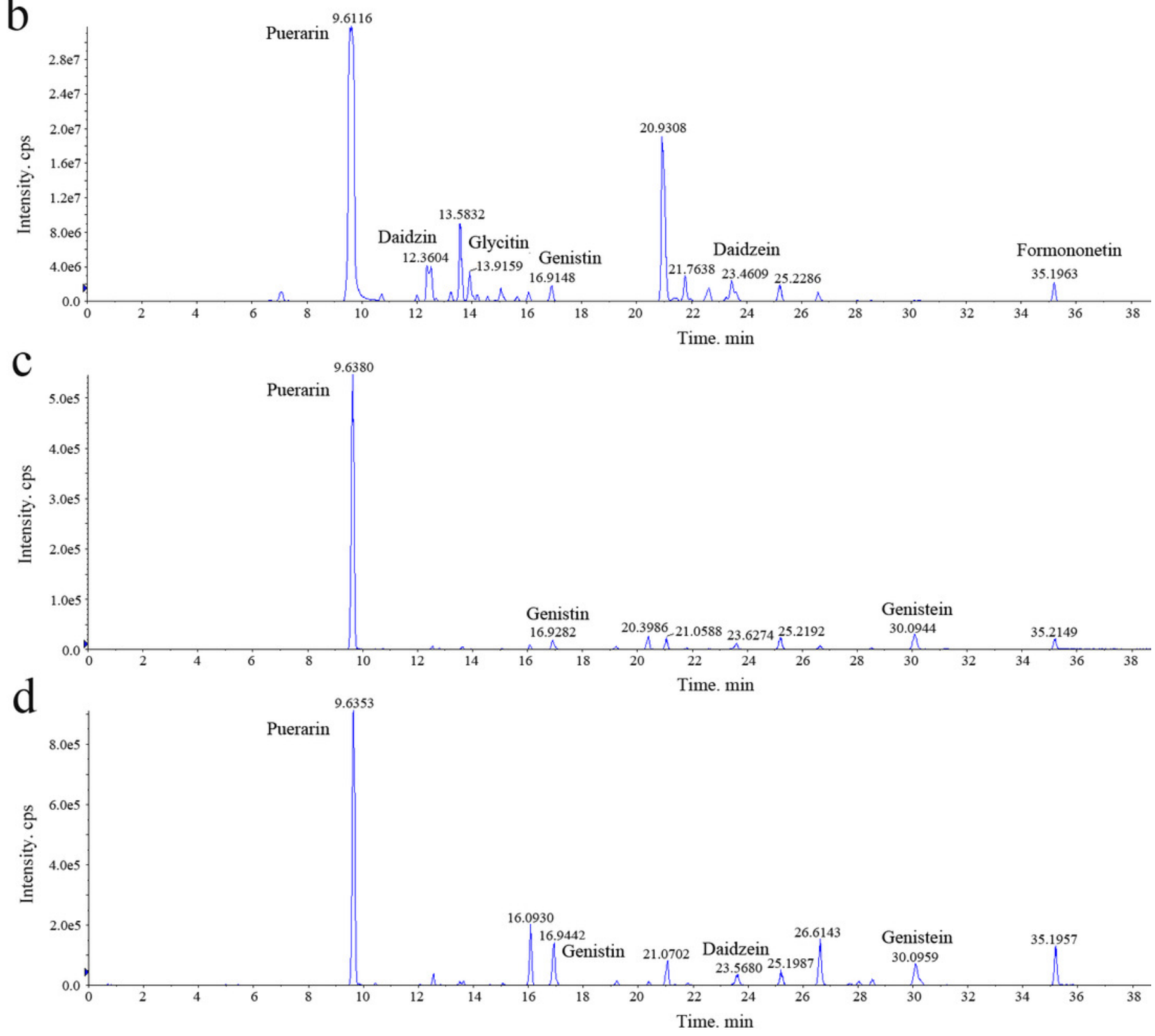
Figure 2

Analysis of expression profiles in Pueraria lobata three tissues (roots, leaves, and stems).

(a) Distribution of number of unigenes having different expression levels in tissues. (b)

Expressed unigenes in tissues depicted as boxplots. Statistical comparisons of the medians were made with the Kruskal-Wallis nonparametric test.
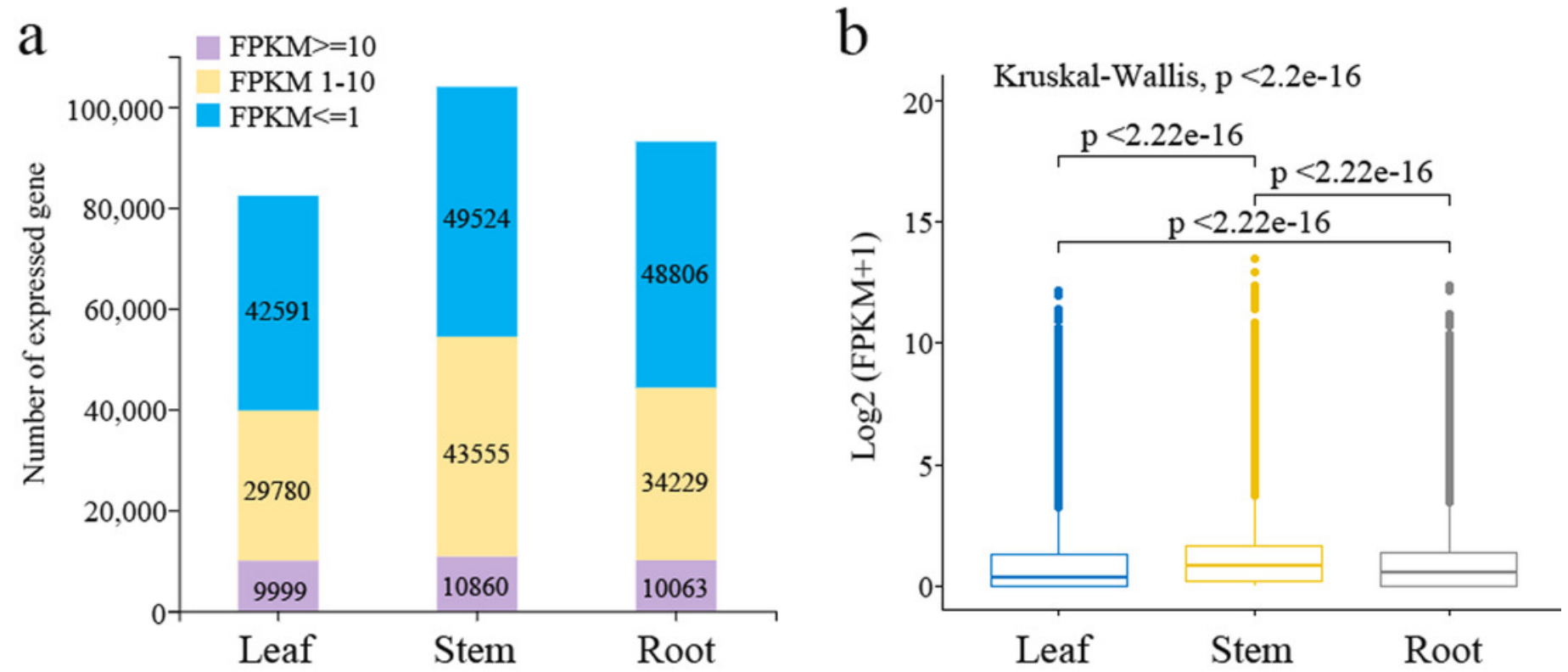
Figure 3

KEGG annotation of Pueraria lobata unigenes.

Number of unigenes that participated in the metabolites' classification (A) and other secondary metabolites' classification (B). 
A

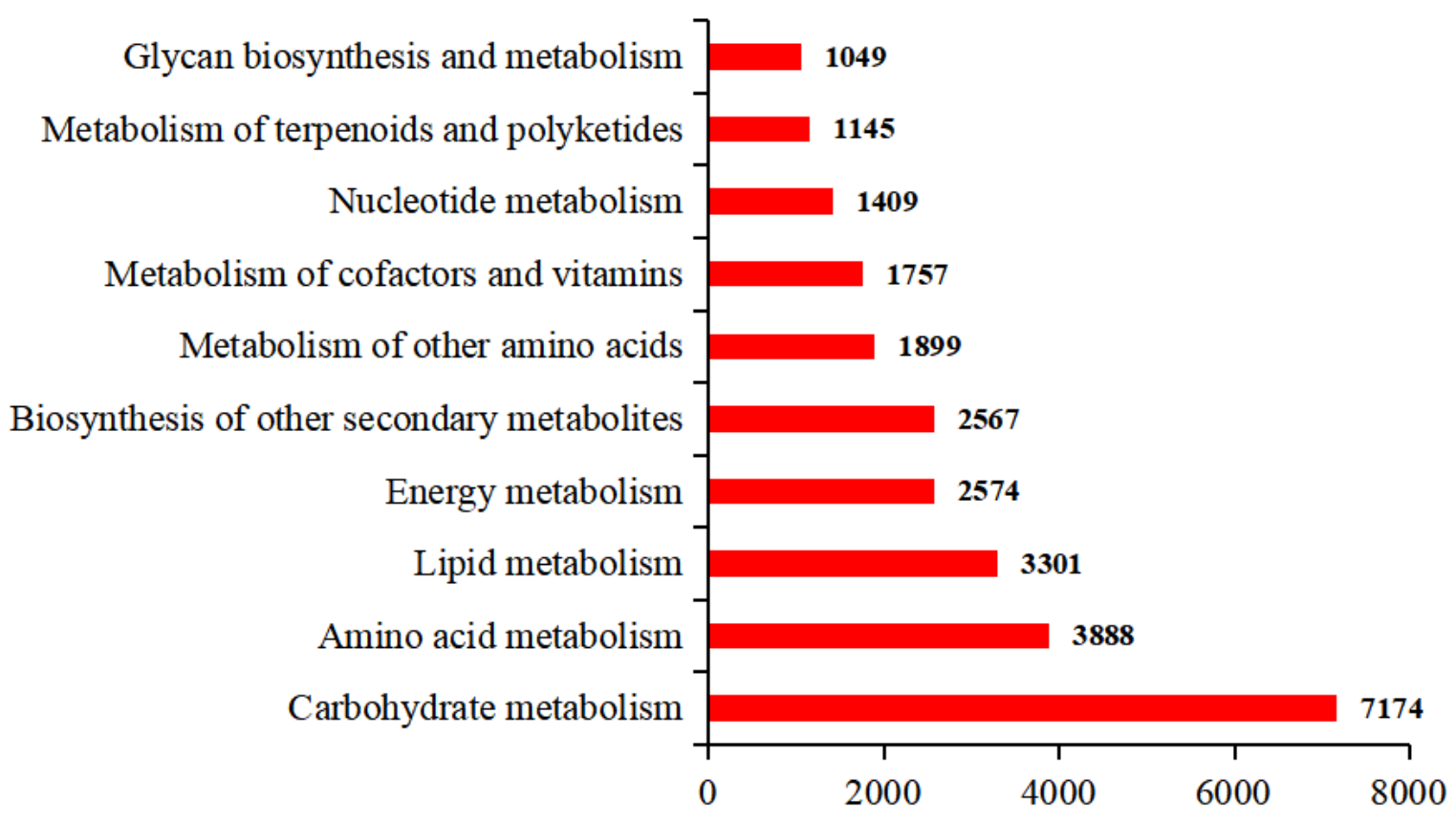

B

Number of unigenes

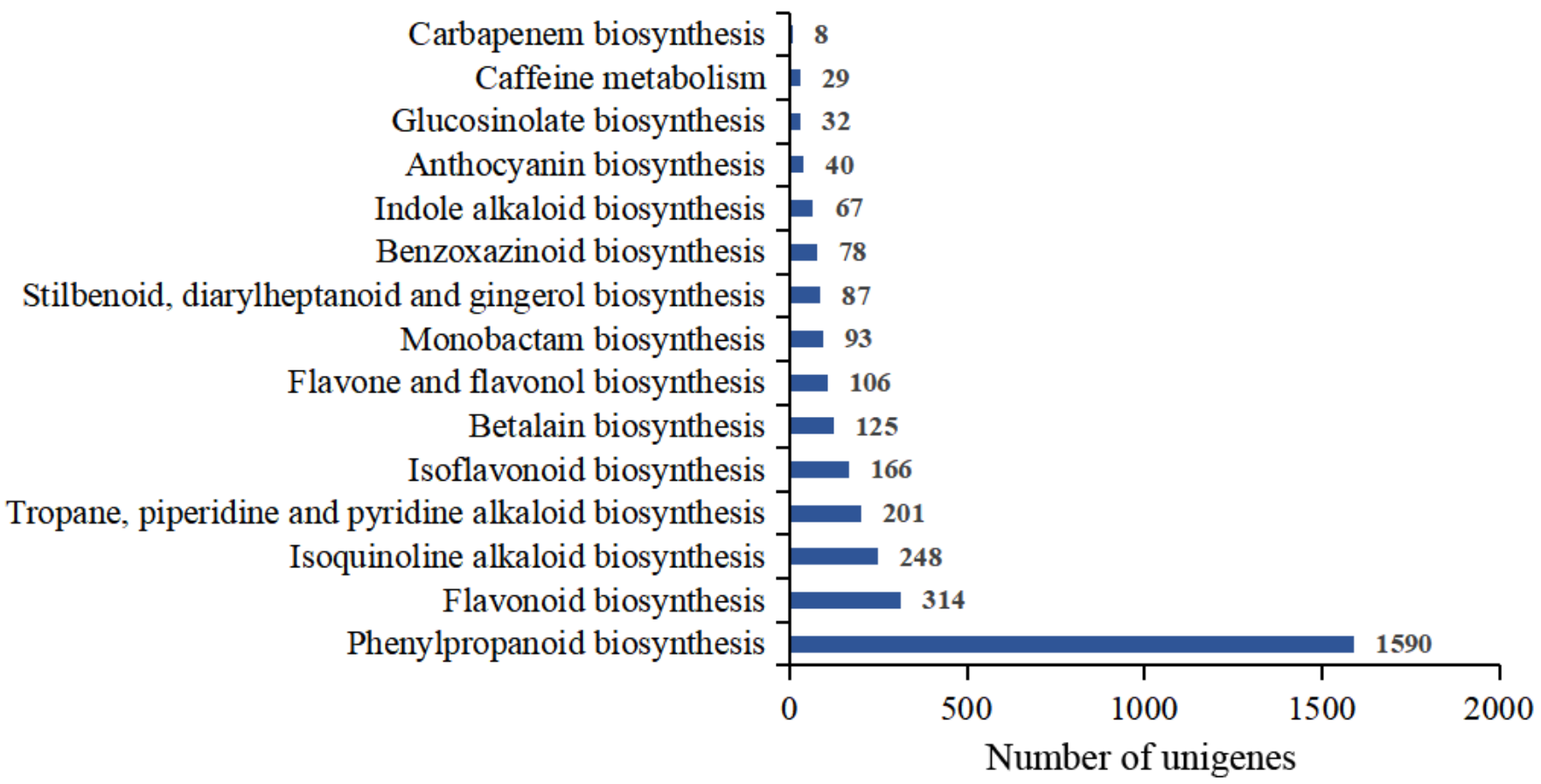




\section{Figure 4}

Predicted pathways for isoflavonoid biosynthesis in Pueraria lobata.

The heatmap shows the expression levels of unigenes encoding enzymes of the key metabolic entry point for the formation of all isoflavonoids. R, roots; L, leaves; S, stems. "Un" and " $\mathrm{CL}$ " represents a cluster of unigenes and transcripts, respectively. Red and blue indicate the high-and low-expression levels, respectively. Different constituents of isoflavonoids are marked in red.

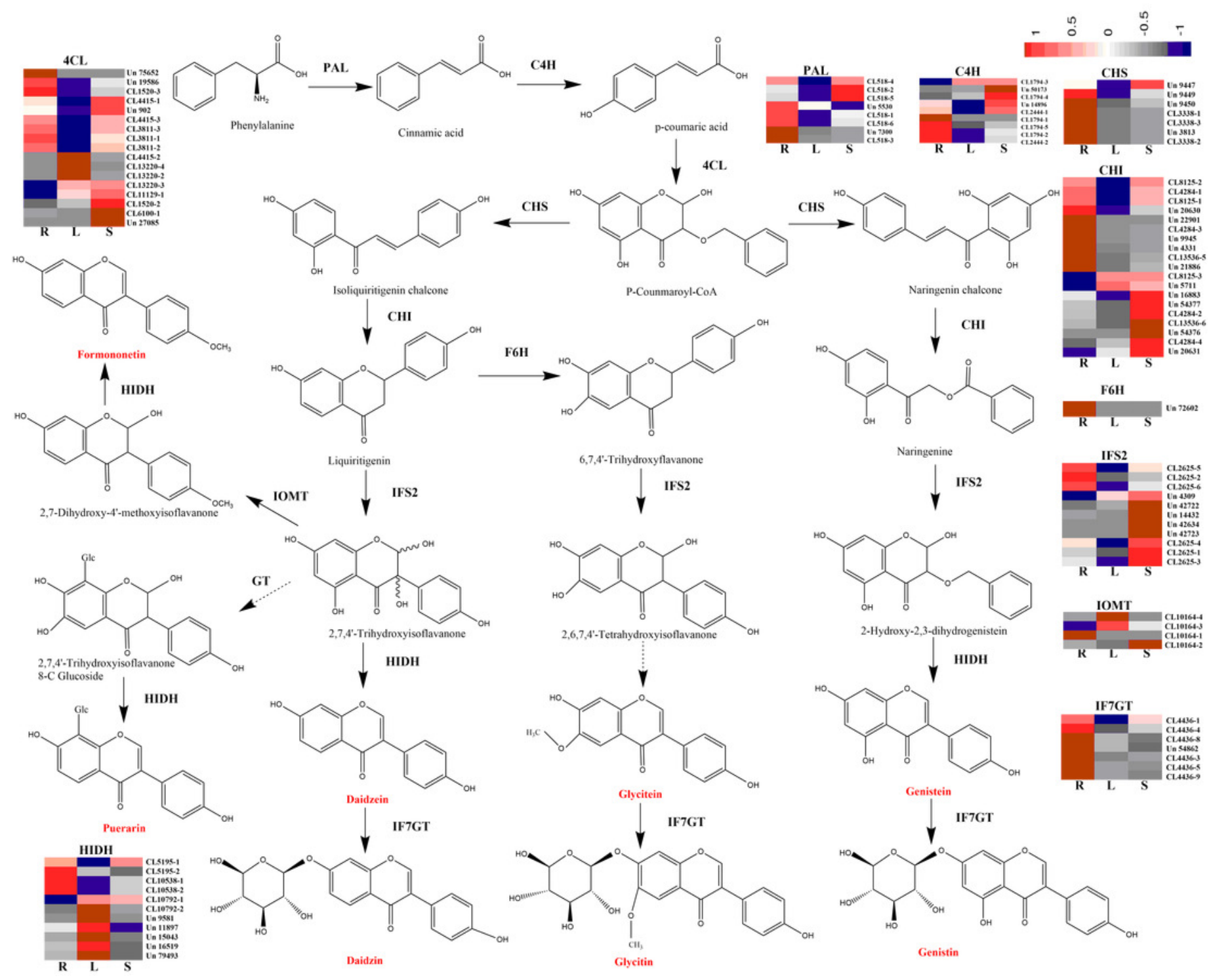




\section{Figure 5}

Multiple sequence alignment and secondary structure of Pueraria lobata IF7GTs with other representative UGTs.

The red-highlighted sections and red letters on a white background respectively represent identical and similar amino acids. A conserved PSPG motif (potential UDP-binding domain) is marked in black box. GMUGT, isoflavone 7-O-glucosyltransferase UGT4 in Glycine max (NP_001304440.2); GSUGT, UDP-glycosyltransferase 1 in Glycine soja (XP_028207064.1); isoflavone 7-0-glucosyltransferase in Pueraria montana var. lobata (AMQ26113.1). 

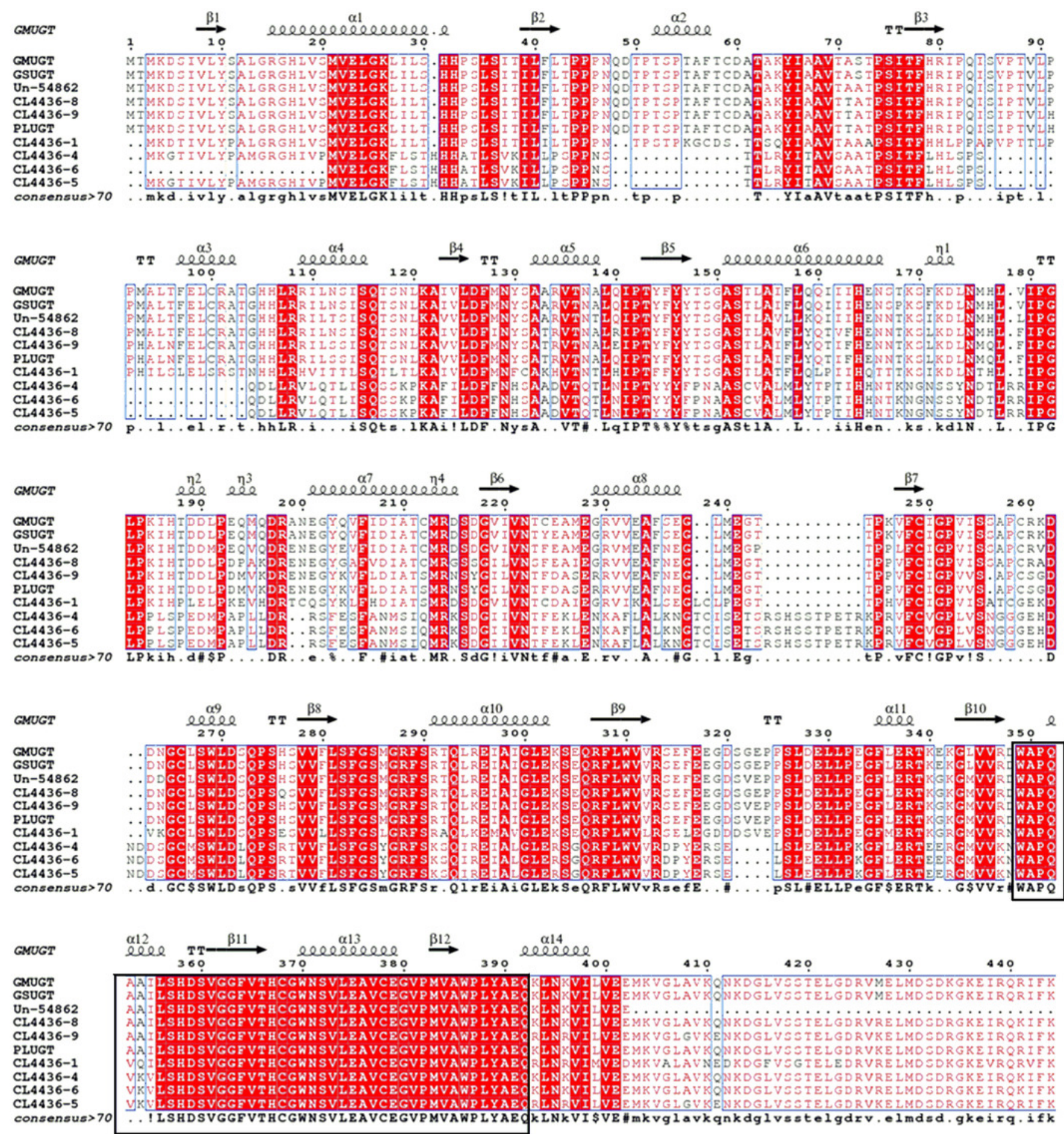

\section{PSPG-box}

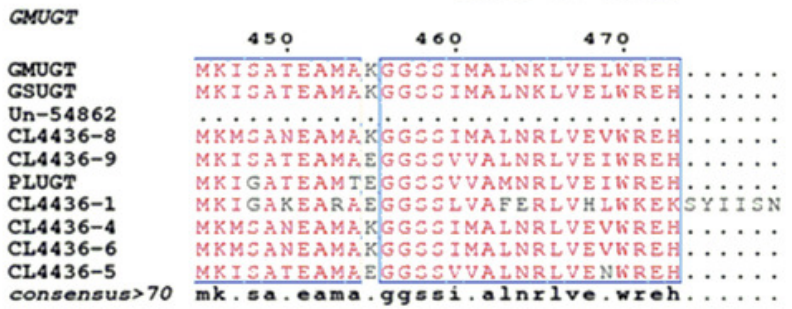




\section{Figure 6}

The qRT-PCR analysis of nine candidate unigenes encoding enzymes involved in isoflavonoid biosynthesis in different tissues of Pueraria lobata.

Relative expression of CL518.Contig3 (PAL), CL2444.Contig2 (C4H), CL1520.Contig3 (4CL), CL3338.Contig1 (CHS), Unigene9945 (CHI), Unigene72602 (F6H), CL2625.Contig2 (IFS2), CL10538.Contig1 (HIDH), Unigene54862 (IF7GT) was normalized with respect to the actin gene (CL10129.Contig5). Blue bars indicate the qRT-PCR results, and red lines show the FPKM values identified via the RNA-Seq analysis. Data is shown as the mean \pm standard error of three replicates. The left $y$-axis is the relative expression level of unigenes obtained by qRTPCR, and the right $y$-axis denotes the FPKM values in the RNA-Seq data.
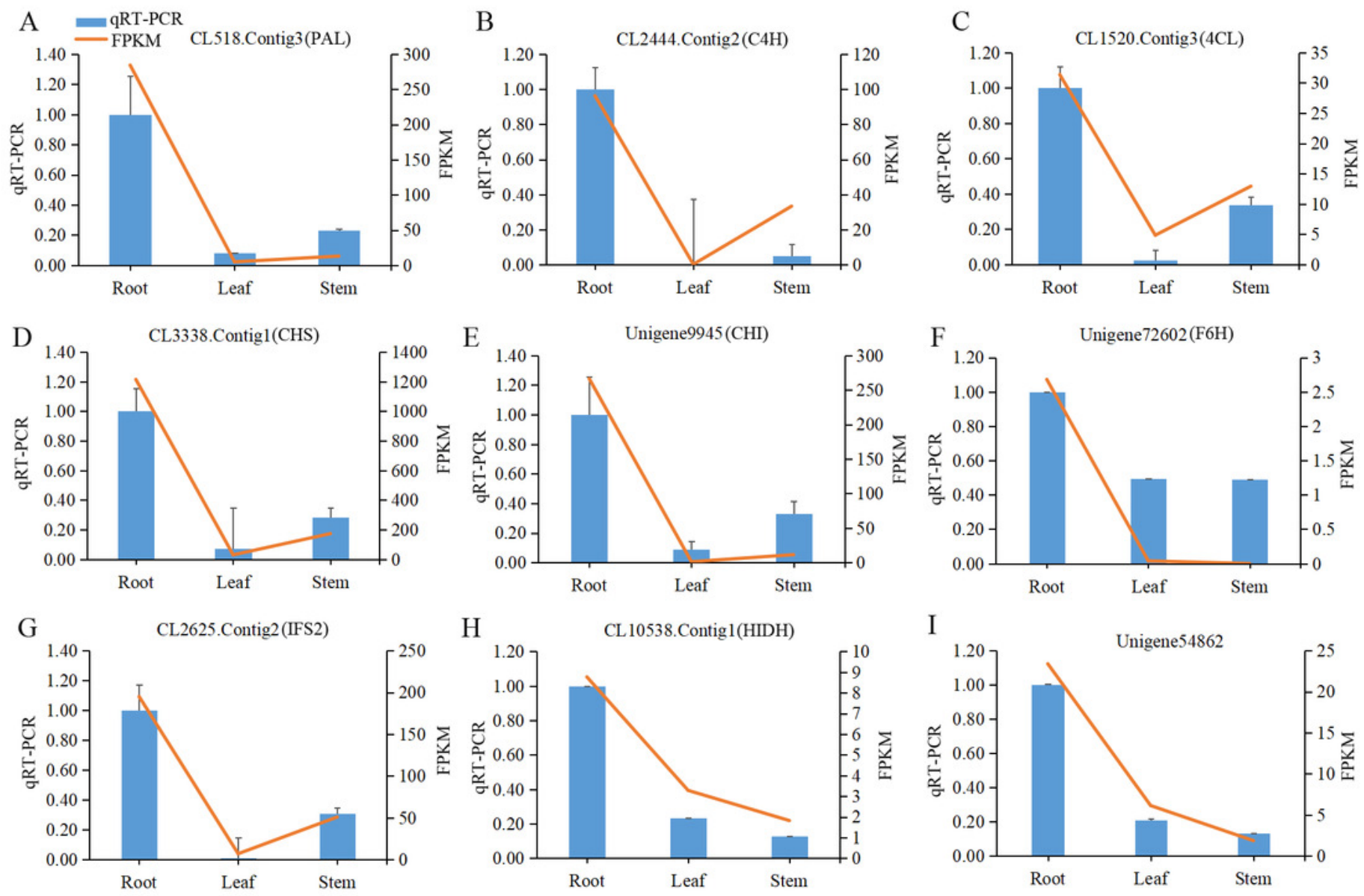


\section{Figure 7}

Expression of unigenes in Pueraria lobata root, stem, and leaf tissues.

(A) Number of unigenes expressed within and among the three tissues summarized in a Venn diagram. (B) The numbers of up- and down-regulated DEGs in root vs. leaf, root vs. stem, root vs. leaf and stem datasets.

A

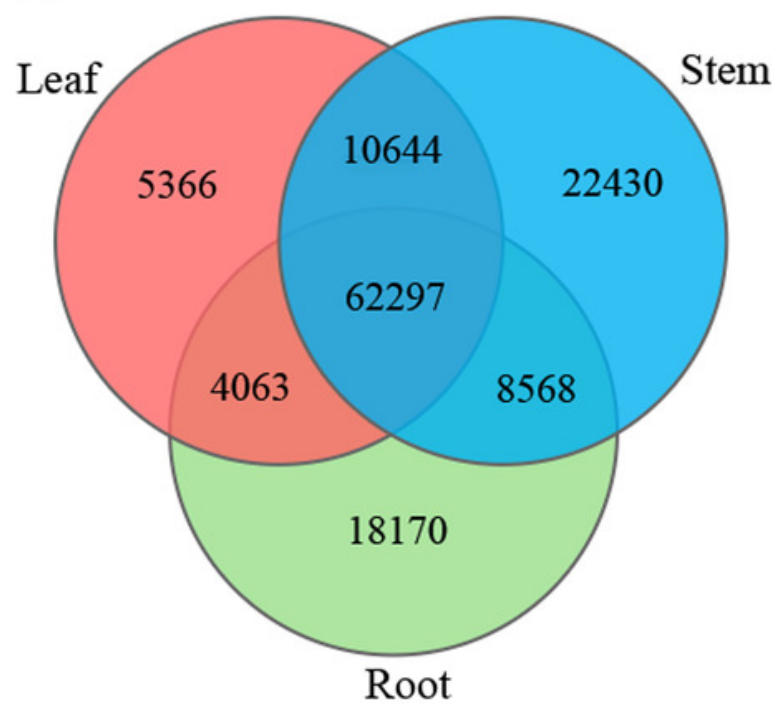

B

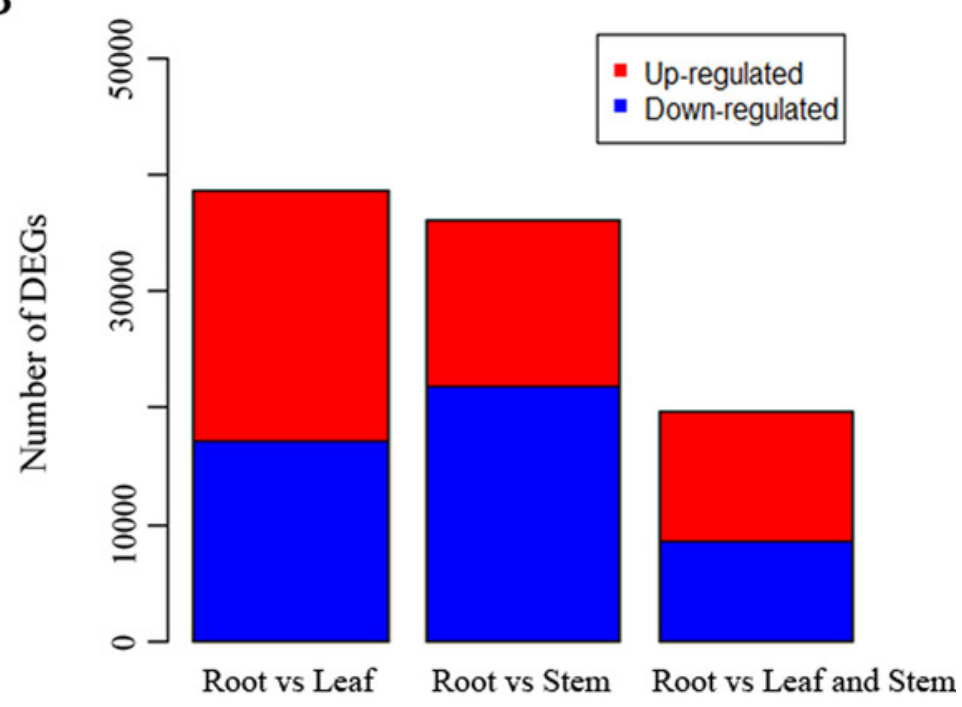


Figure 8

GO and KEGG enrichment analyses ( $A$ and $B$ ) of unigenes expressed specifically in the roots of Pueraria lobata.

\section{GO and KEGG enrichment analyses (A and B) of unigenes expressed specifically in} the roots of Pueraria lobata.
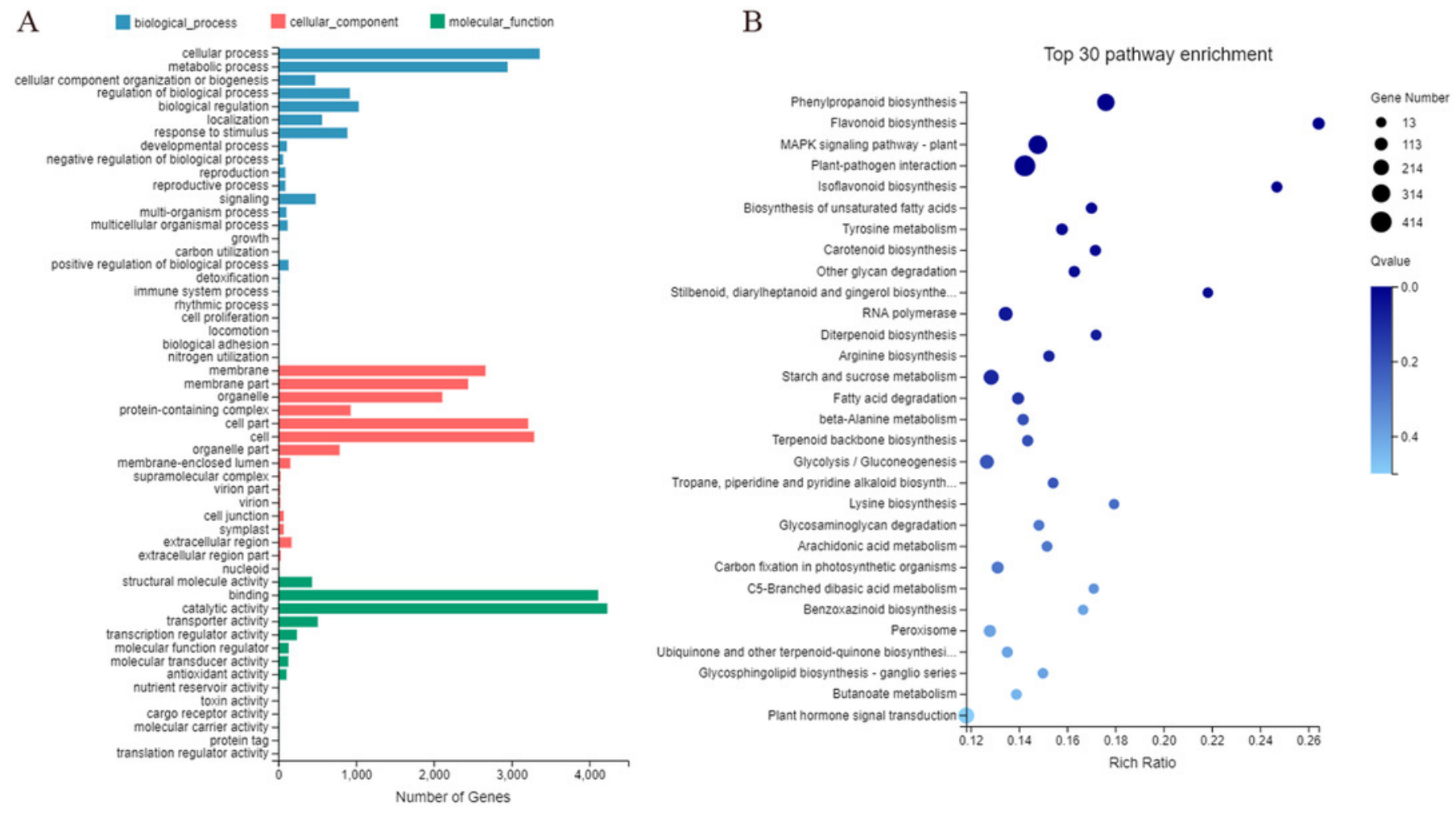


\section{Table 1 (on next page)}

Pueraria lobata unigenes annotated by seven public databases. 
1

2 Table 1 Pueraria lobata unigenes annotated by seven public databases.

\begin{tabular}{lll}
\hline Database & Number annotated & Annotated unigene ratio (\%) \\
\hline NR & 99,480 & 70.60 \\
NT & 81,787 & 58.04 \\
GO & 77,920 & 55.30 \\
KOG & 77,533 & 55.03 \\
KEGG & 75,628 & 53.67 \\
Swissprot & 69,916 & 49.62 \\
Pfam & 66,629 & 47.29 \\
Intersection & 37,498 & 26.61 \\
Overall & 109,687 & 77.84 \\
\hline
\end{tabular}

3 


\section{Table 2 (on next page)}

Summary of isoflavonoid biosynthesis unigenes in three Pueraria lobata tissues. 
1 Table 2 Summary of isoflavonoid biosynthesis unigenes in three Pueraria lobata tissues.

\begin{tabular}{llllll}
\hline $\begin{array}{l}\text { Enzyme } \\
\text { name }\end{array}$ & $\begin{array}{l}\text { EC } \\
\text { number }\end{array}$ & $\begin{array}{l}\text { Unigene } \\
\text { number }\end{array}$ & $\begin{array}{l}\text { No. in } \\
\text { roots }\end{array}$ & $\begin{array}{l}\text { No. in } \\
\text { stems }\end{array}$ & $\begin{array}{l}\text { No. in } \\
\text { leaves }\end{array}$ \\
\hline PAL & 4.3 .1 .24 & 8 & 8 & 8 & 8 \\
C4H & 1.14 .13 .11 & 9 & 9 & 8 & 7 \\
4CL & 6.2 .1 .12 & 17 & 16 & 16 & 15 \\
CHS & 2.3 .1 .74 & 7 & 7 & 7 & 7 \\
CHI & 5.5 .1 .6 & 19 & 17 & 19 & 18 \\
IFS2 & 1.14 .13 .136 & 11 & 8 & 11 & 9 \\
F6H & $1.14 .13 .-$ & 1 & 1 & 0 & 1 \\
IOMT & 2.1 .1 .212 & 4 & 4 & 3 & 4 \\
HIDH & 4.2 .1 .105 & 11 & 10 & 10 & 11 \\
IF7GT & 2.4 .1 .170 & 7 & 7 & 7 & 6 \\
\hline
\end{tabular}

2 EC: Enzyme Commission 


\section{Table 3(on next page)}

Type and number of transcription factor (TF) families encoded via the DEGs (differentially expressed genes) database of Pueraria lobata. 
1 Table 3 Type and number of transcription factor (TF) families encoded via the DEGs (differentially

2 expressed genes) database of Pueraria lobata.

\begin{tabular}{|c|c|c|c|}
\hline TF family & $\begin{array}{l}\text { Number of } \\
\text { unigenes }\end{array}$ & $\begin{array}{l}\text { Up-regulated unigenes } \\
\text { in root vs leaf }\end{array}$ & $\begin{array}{l}\text { Up-regulated unigenes } \\
\text { in root vs stem }\end{array}$ \\
\hline MYB & 375 & 120 & 57 \\
\hline bHLH & 221 & 84 & 44 \\
\hline WRKY & 208 & 66 & 36 \\
\hline AP2-EREBP & 196 & 85 & 39 \\
\hline NAC & 170 & 44 & 32 \\
\hline $\mathrm{C} 3 \mathrm{H}$ & 158 & 48 & 16 \\
\hline $\mathrm{C} 2 \mathrm{H} 2$ & 156 & 48 & 39 \\
\hline G2-like & 101 & 16 & 13 \\
\hline GRAS & 98 & 42 & 11 \\
\hline FAR1 & 93 & 20 & 11 \\
\hline $\mathrm{ARF}$ & 81 & 31 & 15 \\
\hline MADS & 75 & 19 & 12 \\
\hline Trihelix & 75 & 13 & 8 \\
\hline ABI3VP1 & 73 & 21 & 15 \\
\hline C2C2-GATA & 56 & 14 & 10 \\
\hline mTERF & 56 & 18 & 5 \\
\hline C2C2-Dof & 55 & 28 & 4 \\
\hline LOB & 45 & 22 & 10 \\
\hline SBP & 45 & 15 & 7 \\
\hline Tify & 45 & 4 & 8 \\
\hline HSF & 44 & 11 & 9 \\
\hline LIM & 44 & 8 & 8 \\
\hline TCP & 38 & 4 & 1 \\
\hline FHA & 37 & 8 & 3 \\
\hline TIG & 34 & 9 & 4 \\
\hline TUB & 34 & 10 & 4 \\
\hline Zn-clus & 32 & 2 & 2 \\
\hline Alfin-like & 31 & 6 & 1 \\
\hline bZIP & 27 & 4 & 8 \\
\hline BSD & 24 & 3 & 3 \\
\hline RWP-RK & 14 & 4 & 0 \\
\hline zf-HD & 13 & 1 & 0 \\
\hline GeBP & 8 & 2 & 0 \\
\hline Other & 223 & 75 & 39 \\
\hline Total number & 2985 & 905 & 474 \\
\hline
\end{tabular}

3 